\title{
Numerical model of liquid phase mixer
}

\author{
Model numeryczny mieszalnika fazy ciekłej
}

\section{BARTOSZ MOCZULAK WOJCIECH MIASKOWSKI KRZYSZTOF NALEPA JAKUB JASIŃSKI *}

Presented is the course of conduct during modeling of phenomena occurring in a mixer intended for mixing liquids. Issues related to the numerical description of phenomena occurring in this type of constructions are discussed, the method of modeling the mixing process for one type of liquid by the analysis of several types of mixing arms is presented and the results of simulation research are discussed.

KEYWORDS: mixing tank, simulation, CFD

Mixing is a process that aims to achieve a homogeneous structure in a multi-phase environment. The mixture does not allow transfer of heat and/or mass between one or more streams, components or phases [1, 2].

The selection of an appropriate mixer requires knowledge of many issues related to mixing theory, including degree of mixing, efficiency and mixing intensity [3]. A good solution when designing mixers is the use of numerical analysis, which allows you to analyze many configurations at relatively low costs.

The aim of the study is to develop a simplified numerical model of a mixer that will allow for the initial assessment of the possibilities of various rotor configurations in relation to the approximate mixing process.

The article was based on a question from the industrial sector, which concerned the creation of a simplified model of a numerical mixer based on commercial programs. The simplified numerical model is to be used to evaluate the work and changes occurring in the mixing process, while maintaining the possibilities of various configurations of the mixer's structure and the geometry of the tank.

The experimental and research work will be carried out by the staff employed in the plant, with different qualifications, which determined the level of sophistication and complexity of the numerical model and the choice of software used in the company.

* Mgr inż. Bartosz Moczulak (bartosz.moczulak@uwm.edu.pl), dr inż. Wojciech Miąskowski (wojmek@uwm.edu.pl), dr inż. Krzysztof Nalepa (nalepka@uwm.edu.pl), inż. Jakub Jasiński (jasiorrro@gmail.com) - Wydział Nauk Technicznych Uniwersytetu Warmińsko-Mazurskiego

\section{Numerical analysis}

During the numerical analysis of the mixer, the geometry shown in fig. 1 was used. It consists of a mixer shaft mounted concentrically to the axis of the tank and baffles mounted on the inner walls of the tank, whose main task is to eliminate the formation of the funnel during mixing.

As a preliminary assumption an analysis of individual configurations for the mixing process of one liquid phase (in this case water) was adopted. The rotor configurations referred to different types of blades and number of revolutions to allow the estimation of the impact of geometry changes of the rotor and rotational speed on the mixing process (fig. 1 and fig. 2).
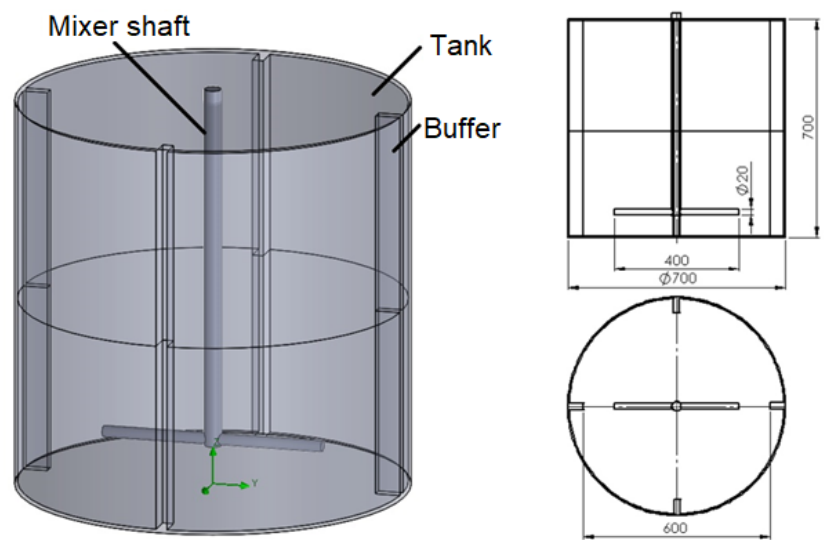

Fig. 1. Geometric model of the mixer with general dimensions

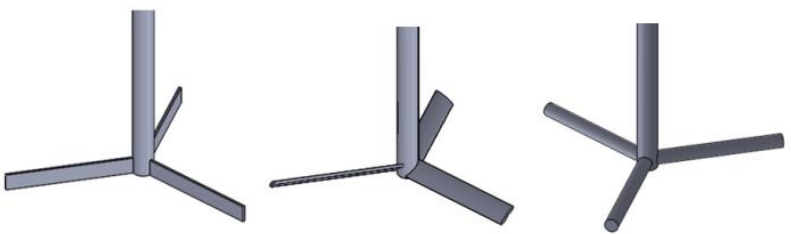

Fig. 2. Three types of agitator rotors (from the left): turbine blend, propeller agitator, agitator with roller blades 


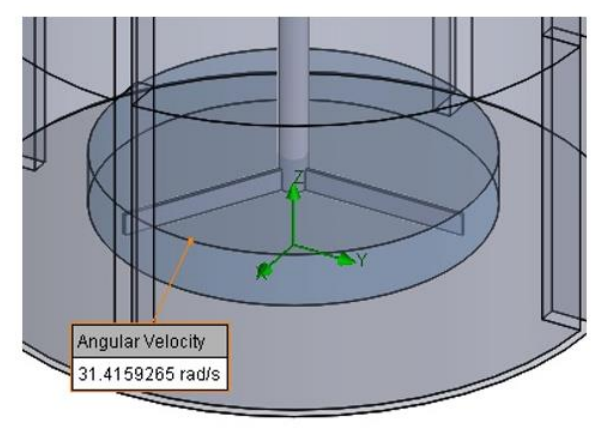

Fig. 3. Rotational region used in the simulation

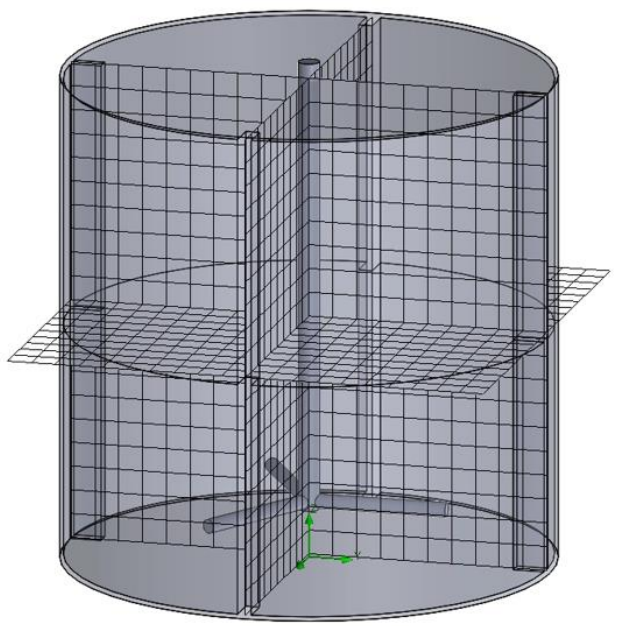

Fig. 4. Grid used in the study
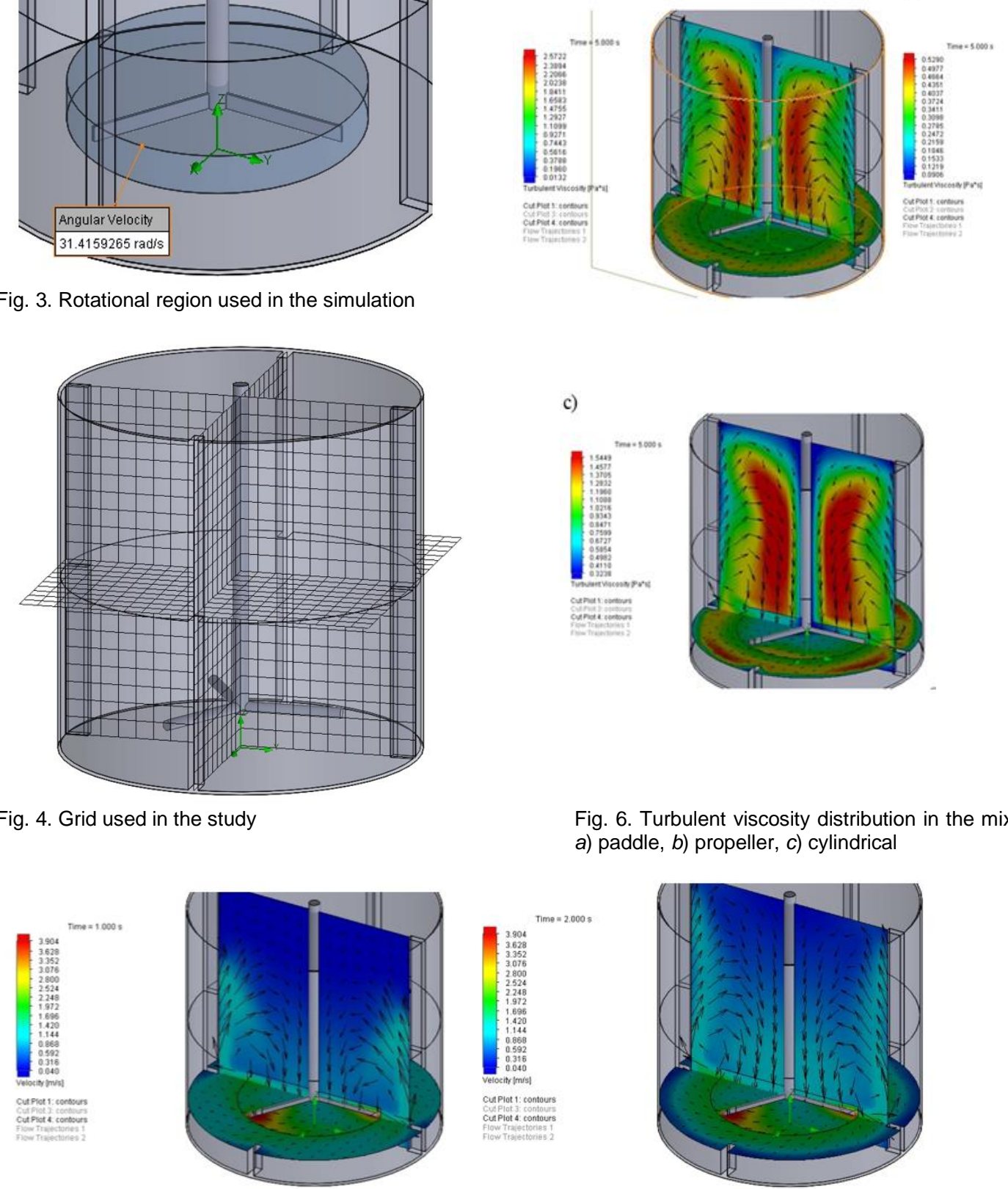

b)

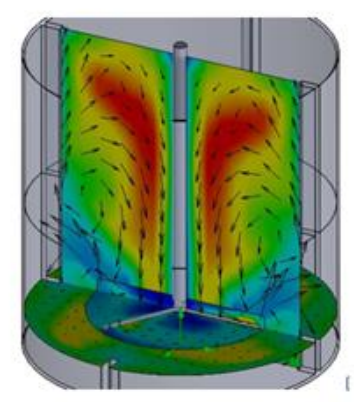

Fig. 6. Turbulent viscosity distribution in the mixer at $300 \mathrm{rpm}$ for the rotor: a) paddle, b) propeller, c) cylindrical
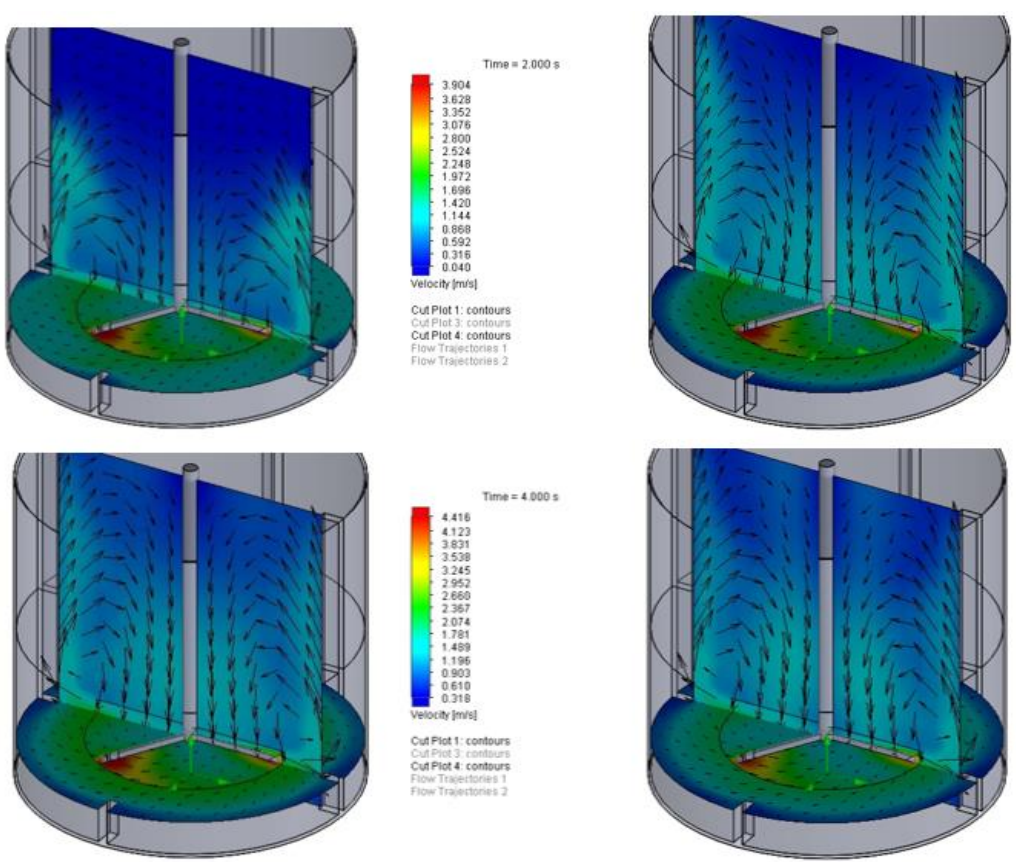

Fig. 5. Distribution of liquid velocity in a mixer with a propeller rotor for $300 \mathrm{rpm}$ in subsequent analysis seconds 
a)

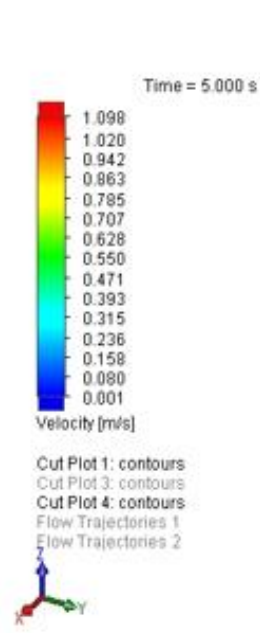

b)
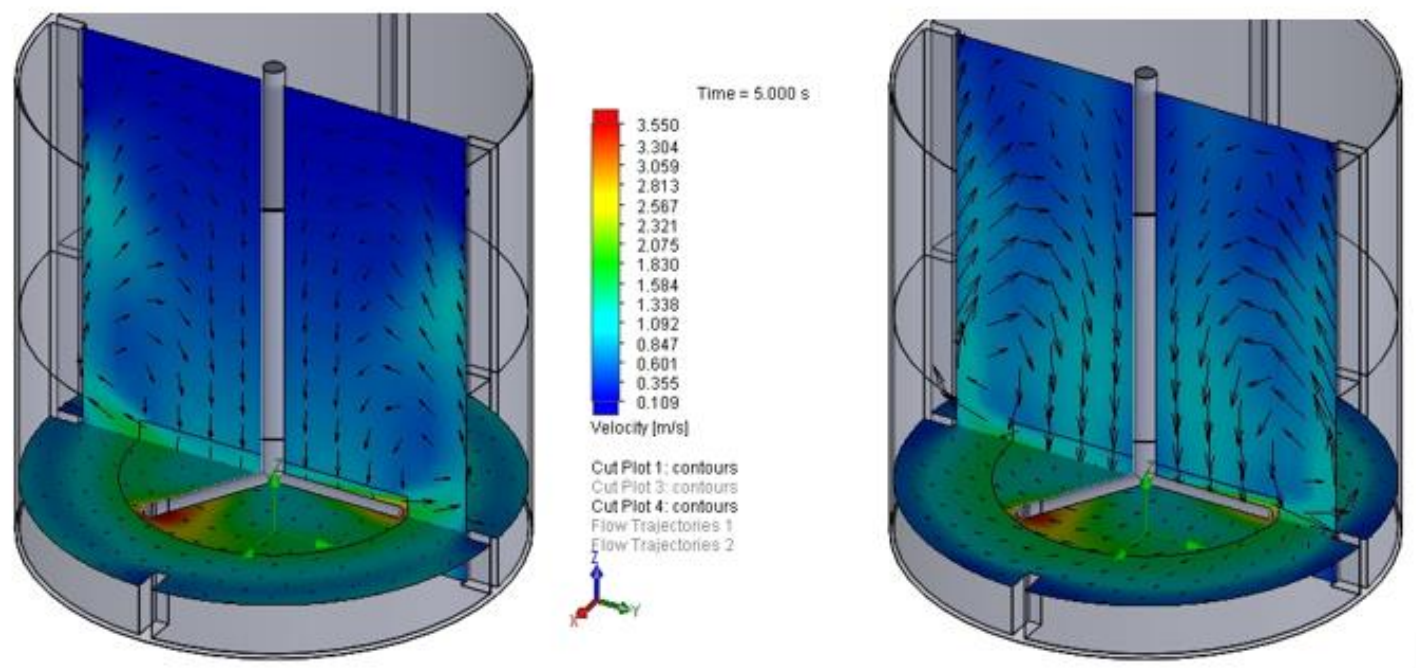

Fig. 7. Fluid velocity distribution in the axis plane and the plane of the roller rotor for velocity: a) $100 \mathrm{rpm}$, b) $300 \mathrm{rpm}$

The numerical analysis of the mixer was carried out in a Solid Works environment with the addition of Flow Simulation. During the analysis, the internal flow calculation mode was adopted, taking into account gravity and time. The calculations assumed:

- mixing time $5 \mathrm{~s}$ and time step $0.1 \mathrm{~s}$,

- boundary conditions: pressure in the tank $101325 \mathrm{~Pa}$, temperature $273 \mathrm{~K}$.

Due to the rotating element, it was decided to use the Rotating Regions option, which makes it possible to reflect the rotating impeller in the model [4] (fig. 3).

The mesh used is shown in fig. 4 . In order to simplify the model as a medium in the computational domain, a homogeneous phase (water) was assumed. As a criterion for the assessment of changes resulting from rotation of the centrifuge, it was assumed to display the results of the velocity distribution of the fluid and the turbulent distribution of the viscosity of the medium, which in a selected manner is shown in figs. $5-7$.

\section{Results of computer simulation}

In the first place, the focus was on the fluid velocity distribution for one type of rotor and one rotational speed. The results are summarized in fig. 5 - every 1 second, respectively. $300 \mathrm{rpm}$ was adopted as the rotational speed. Distribution of the speed of propagation of the liquid takes the form shown in fig. 5 . It is visible that the so-called whirls during mixing.

Fig. 6 presents examples of results showing turbulent viscosity distribution in a mixer at $300 \mathrm{rpm}$ for selected types of rotor.

The turbulent viscosities of the fluid take the form consistent with the predictions. It is possible to observe clear differences in the viscosity distributions for selected rotors, which proves the correct character of the calculations performed, taking into account the influence of geometry on the nature of the medium flow.

Fig. 7 presents the results showing the velocity distribution of the mixing fluid for the selected rotor at different rotational speeds. It is possible to observe a marked increase in the velocity of the mixed medium, visible in diagrams showing both distribution and velocity values.

\section{Conclusions}

Based on the selected results of the mixing simulation, it can be concluded that the simplified mixer model takes into account the change of the rotor geometry and the change of the shaft rotational speed. The observed differences in changes in the viscosity and speed distributions of the agent indicate that the simulation conditions have been correctly adopted. The article is limited to the presentation of selected speed distributions for exemplary mixer geometries and rotational speeds.

Presented numerical model of the agitator allows in a simplified, fast and understandable way for the target team of designers to verify the introduced structural changes in the construction and operation principle of the mixer. The choice of the computing environment allows for simulation of flow and simultaneous creation of the necessary technical documentation of individual components. Due to the access to many simulation settings, the target group of recipients has the possibility to conduct a numerical simulation for media with various physical properties.

The next stage of research will be the validation of computer simulation results on the test stand and increasing the functionality of the numerical model by allowing the simulation of the flow of two and/or multi-phase factors with different physical properties, e.g. two liquid phases or a liquid phase and body pellets solids.

\section{REFERENCES}

1. Stręk F. „Mieszanie i mieszalniki”. Warszawa: WNT, 1981. 2. Shao T., Hu Y., Wang W., Jin Y., Cheng Y. "Simulation of solid suspension in a stirred tank using CFD-DEM coupled approach". Chinese Journal of Chemical Engineering. 2013.

3. https://mech.pg.edu.pl/documents/4555684/4565480/mc.pdf (access: 30.03.2018).

4. SolidWorks Users' Guide.

Translation of scientific articles, their computer composition and publishing them on the website www.mechanik.media.pl by original articles in Polish is a task financed from the funds of the Ministry of Science and Higher Education designated for dissemination of science. 